\title{
Near infrared reflectance measurement of nitrogen faecal losses
}

\author{
L Benini, S Caliari, F Bonfante, G C Guidi, M T Brentegani, G Castellani, C Sembenini, \\ E Bardelli, I Vantini
}

\begin{abstract}
Chemical methods of measuring nitrogen in stools are complex, unpleasant, and therefore rarely performed. Recently, near infrared reflectance (NIRA) has been suggested for stool analysis. The aim of this study was to evaluate the possible application of this method in routine faecal nitrogen measurement. Nitrogen concentration and daily output were measured in the stools of 83 patients using NIRA and, for comparison, the Kjeldahl method. Nitrogen concentration and output ranged between $0.4-2.72 \mathrm{~g} \%$ and $0.45-8.96$ g/day respectively. Correlation coefficients $(r)$, of 0.89 and 0.97 were found between the two methods for concentration and output respectively, and similar values were found in patients on enteral nutrition. Repeated measurements from the same stool collection, requiring only a few minutes, allowed homogenisation to be avoided. NIRA seems to be an easy, fast, and reliable alternative to chemical assays of nitrogen measurement in the management of patients with digestive disorders.
\end{abstract}

The evaluation of nitrogen balance is essential in the management of patients with nutritional problems. When absorption deficits are not present only urinary nitrogen is usually measured, since a reliable value can be attributed to other losses (skin, stools, secretions). This approach, however, may lead to severe underestimation of protein imbalance in gastrointestinal diseases causing malabsorption or gastrointestinal protein losses.' The quantification of faecal protein loss is therefore necessary in these diseases. Chemical methods are so unpleasant and time consuming that they are rarely used - not only in clinical practice but also in research studies on protein balance. ${ }^{23}$ Recently, near infrared reflectance analysis (NIRA) has been introduced in clinical chemistry for macronutrient measurement in faeces. ${ }^{+5}$ It is based on the measurement of the radiations in the infrared spectrum close to the visible $(700-2500 \mathrm{~nm})$ scattered by the surface of a sample. ${ }^{6}$ The scattered component is inversely correlated to the energy absorbed at each wavelength by specific chemical bonds. The method is very accurate for measurements in homogenous materials, and is widely used in the pharmaceutical and food industries. In composite materials such as faeces, interfering absorbance can be caused by the other constituents, and this makes calibration more complex. ${ }^{7}$ This study aimed to evaluate the analytic capacity of NIRA in the measurement of faecal nitrogen in patients with digestive diseases who were either taking a normal hospital diet or were on enteral nutrition.

\section{Methods}

\section{PATIENTS}

We studied the faeces of 83 patients admitted to our department, which is a referral centre for chronic gastrointestinal diseases, in the period January 1988-November 1990. These patients had come into hospital for suspected malabsorption or severe digestive problems and their diagnoses are summarised in the Table. Most chronic pancreatitis patients were admitted as part of a long term study of the natural history of the disease. In six patients (mesenteric infarction (3), severe Crohn's disease (2), radiation enteritis (1)) short bowel syndrome was present. The coeliac disease patients were studied before they were put on a gluten free diet. Patients with radiation enteritis (colonic (2), bladder (1), uterine (1) cancers) were studied for pain or subocclusive symptoms; no tumour spread was evident. The two patients with pharyngeal dysphagia secondary to central nervous system disease were sent to us for swallowing problems requiring prolonged enteral nutrition after brain stem operations. In nine patients (extensive Crohn's disease (2), intestinal resection (2), radiation enteritis (1), pharyngeal dysphagia (2), severe pancreatic insufficiency (2)) 33 stool samples were studied while they were on different enteral nutrition regimens (polymeric or elemental). The others were on a standard hospital diet $(70-80 \mathrm{~g}$ of protein/day).

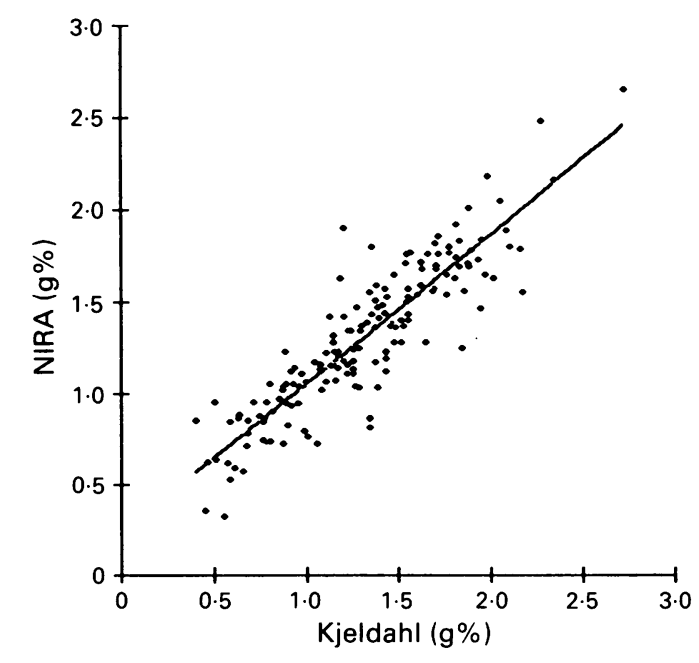

Figure 1: Correlation between faecal nitrogen concentration $(\mathrm{g} \%)$ measured by the mineralometric method (Kjeldahl) and near infrared reflectance analysis $(N I R A)\left(y=0 \cdot 24+0 \cdot 82^{\star} x\right.$. $S_{\mathrm{y} / \mathrm{x}}=0 \cdot 18, r=0 \cdot 893$ ) 


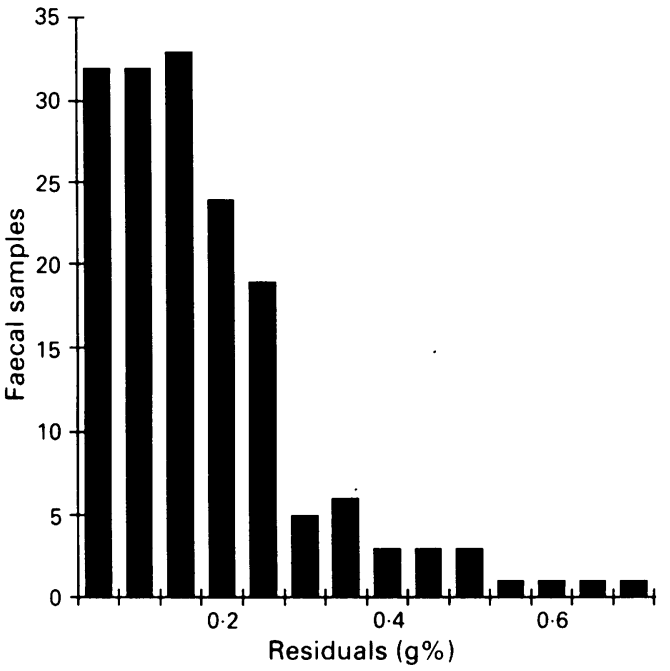

Figure 2: Frequency distribution of differences between the results of the reflectometric (NIRA) and mineralometric (Kjeldahl) methods of nitrogen measurement in our faecal samples.

STOOL ANALYSIS

The stools were collected on three consecutive days after withdrawal of all drugs that might interfere with intestinal transit or absorption. Nitrogen output was measured for clinical purposes in the stools from all three days, but only the stools of the first day were used to calculate the correlation between the two methods. Most patients were repeatedly admitted to hospital or were put on different nutritional regimens during a lengthy admission (for example, before and after ileal resection for Crohn's fistulae). Repeated nitrogen measurements were performed in these patients on the assumption that there would be a substantial change in stool characteristics. In total, 163 faecal samples were considered (median, 2 samples per patient; range $1-5)$.

\section{ANALYTICAL METHODS}

\section{NIRA method}

For the NIRA, a small faecal sample (2-3 g) was pressed between a glass slide and a plastic cup to yield a smooth surface and a constant volume. The cup was then inserted in a drawer of the recording equipment (InfraAlyzer 450, BranLuebbe, Rome) for automatic reading of the reflectance at 19 different wavelengths in the infrared spectrum. For the nitrogen measurement, the instrument had first to be calibrated by a multilinear regression derived equation to correlate the logarithms of the reflectances with the results of the Kjeldahl method. This was done in 87 stool samples (not considered for the assessment of the accuracy of the method), chosen for a nitrogen content covering the range found in our clinical practice $(0 \cdot 4-2 \cdot 7 \%)$, with a sufficient number of samples for each concentration to minimise interferences from the stool matrix. The six filters providing the best fitting readings and their correlation coefficients were then used ('calibration curve') for nitrogen measurement. The coefficients of variation $(\mathrm{CV})$ of the NIRA were calculated both from 25

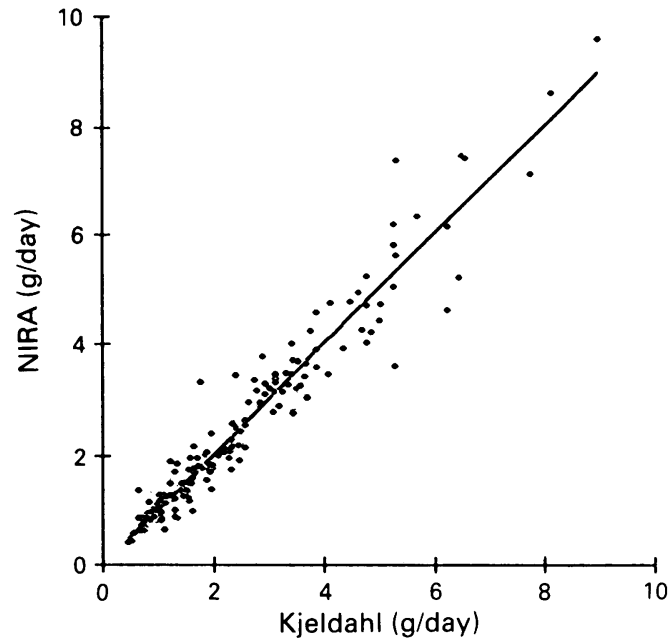

Figure 3: Correlation between faecal nitrogen excretion ( $g$ ) day) measured using the mineralometric (Kjeldahl) and the reflectometric (NIRA) methods $\left(y=0.02+1 \cdot 004^{\star} x . S_{\mathrm{v} / \mathrm{x}}=\right.$ $0.45, r=0.97)$.

repeated measurements on two faecal samples with high and low nitrogen content (within run $\mathrm{CV}$ ) and from three repeated measurements on 16 samples (between run $\mathrm{CV}$ ).

\section{Kjeldahl method}

The Kjeldahl mineralometric method ${ }^{\mathrm{x}}$ was used as reference both for the calibration of the InfraAlyzer and to assess the accuracy of nitrogen measurement by the NIRA method. It is based on strong acid digestion of all nitrogen contents of $1 \mathrm{~g}$ of stools (by prolonged boiling with $30 \mathrm{ml}$ concentrated sulphuric acid) to yield $\mathrm{NH}_{4}{ }^{+}$. Ammonia is thereafter distilled by boiling with $100 \mathrm{ml}$ of $32 \% \mathrm{NaOH}$, collected in boric acid, and titrated with $0.1 \mathrm{~N} \mathrm{NaOH}$. This was done using a semiautomatic equipment (Büchi 430 and 321, Flawil, Switzerland) and an automatic titration system (RTS 822, Radiometer, Copenhagen). A mean CV of $5 \cdot 8 \%$ was obtained

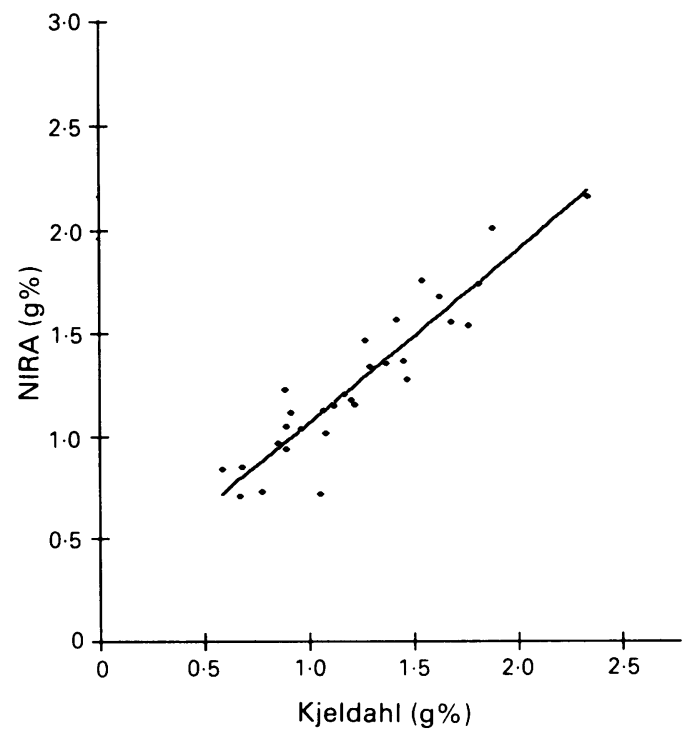

Figure 4: Correlation between faecal nitrogen concentration measured using the mineralometric (Kjeldahl) and the reflectometric (NIRA) methods in patients on enteral nutrition $\left(y=0 \cdot 23+0 \cdot 84^{\star} x . S_{\mathrm{v} / \mathrm{x}}=0 \cdot 14, r=0.93\right)$. 
with the mineralometric method from eight repeated measurements on two samples.

STATISTICAL METHODS

The APC program (Bran-Luebee) was used for multiple regression. It allows automatic reflectance data acquisition from the InfraAlyzer, and analysis of the residuals between expected (NIRA) and observed (Kjeldahl) values. The Pearson's coefficient ' $r$ ' was used to evaluate the correlation both between the results of the two methods and between residuals and nitrogen concentration.

\section{Results}

The median nitrogen concentration we found was $1 \cdot 28 \mathrm{~g} \%$ (range $0 \cdot 32-2 \cdot 65$ ) using the NIRA and $1 \cdot 29 \mathrm{~g} \%(0 \cdot 4-2 \cdot 72)$ using the mineralometric method. The daily faecal losses using the two methods were 1.92 (0.41-9.60) and 1.93 (0.45$8 \cdot 96)$ g/day respectively.

Repeated NIRA measurements on the same stool sample yielded a within run $\mathrm{CV}$ of $5 \cdot 5 \%$ and a between run $\mathrm{CV}$ of $5 \%$. The mean of five repeated NIRA measurements from different parts of the stool collection before homogenisation always gave a result within $\pm 10 \%$ of the final value.

The correlation between the concentrations measured by the mineralometric and the reflectometric methods is shown in Figure 1. A correlation coefficient of 0.89 is found. The residual values (differences between results obtained using the two methods) are not correlated with the nitrogen content $(r=0 \cdot 10, N S)$. The absolute difference between the nitrogen concentrations calculated by the two methods was mean (SEM) $0.15(0.01)$ g\% (Fig 2). The highest residual values were found in liquid or in acholic samples. A much better correlation was found between the daily outputs calculated by the two methods ( $r=0.97$ ) (Fig 3), since for their calculation the concentrations are multiplied by the same faecal weight.

A similar relation between the two methods was found in patients on enteral nutrition (Fig 4). This suggests that the dietary nitrogen source is not important for the reflectometric properties of stools, and that stool nitrogen can be measured in people on different diets with the same calibration parameters.

\section{Discussion}

Protein malabsorption is a late finding in many gastrointestinal diseases, and is therefore of little value in diagnosis. ${ }^{9}$ However, its quantitation is essential for the management of patients with nutritional problems. ${ }^{10-12}$ Traditional methods of nitrogen measurement are complex, time consuming, and rather dangerous and therefore faecal nitrogen losses are rarely measured in clinical practice.

NIRA could represent a useful alternative method. In fact, it is simple, minimises faecal handling, and can therefore be used in daily practice. Nurses can easily be instructed to obtain the data after each bowel movement and without storing the faeces. The method also avoids stool homogenisation, allowing repeated measurements from different parts of the daily collection.

The assay is based on the measurement of the scattered infrared light reflected by a sample. The concentration of the substance to be studied is automatically calculated from these readings using the wavelengths and the coefficients obtained during the calibration process by a multiparametric regression. This means that the reflectometric results cannot be 'more accurate' than the reference method, which is the ideal target, and that the results will always represent an approximation because of interference caused by other physical and chemical properties of the stools. We can only improve accuracy by using stool samples with different characteristics for calibration. The nitrogen concentration and the 'interfering' characteristics of the samples used for the calibration will in fact determine which stools are accurately measurable. Samples with different characteristics cannot be produced in vitro by adding nitrogen compounds to normal stools (this causes an abnormal reflectance), and this explains the need to obtain stools from patients with gastrointestinal disorders both for the calibration process and for evaluating the accuracy of the method. The calibration curve we obtained proved to work in all the patients studied for the last three years in our department, over a range of nitrogen concentration wider than that previously reported.+ The results obtained show that NIRA, if perhaps not accurate enough for nitrogen measurement in some research studies, provides an adequate evaluation of nitrogen malabsorption in daily clinical practice. The difference between reflectometric and chemical values shown in Figure 2 can be reduced by using calibration curves specific for liquid or acholic stools. We consider that the scattering of values around the ideal regression line is partly explained by the imprecision of the reference method too. In fact, even if we are aware that an assumption of regression analysis is that errors of the independent variable are small compared with errors of the dependent variable, ${ }^{7}$ this is probably not the case for nitrogen measurement, since both methods gave similar CVs (within run CVs of 5.5 and $5 \cdot 8 \%$ for the NIRA and the mineralometric method respectively). It could be that using a different reference method for nitrogen measurement - for example chemiluminescence analysis ${ }^{13}$ - would result in a better correspondence with the NIRA, but the Kjeldahl method is at present the most widely accepted assay.

The rather expensive equipment (in Italy about $£ 20000$ ) must be balanced against the low running costs and with ability to measure both fat and nitrogen faecal losses with minimal faecal handling and in minimal time.

In conclusion, NIRA is an easy and fairly accurate method of faecal nitrogen assay which would be useful in gastrointestinal and nutritional centres for the daily management of patients with malabsorption.

We wish to thank all the nurse staff of our department for their helpful cooperation and Mrs Carol Thomas-Bulighin for reviewing the English. Some of these data were presented at the 9th 
World Congress of Gastroenterology, Sydney, 26-31 August 1990 (Abstracts I; 521), and at the BSG spring meeting, Warwick, 2830 March 1990 (Gut 1990, 31:A612). This work was supported by the CNR targeted project 'Prevention and Control of Disease Factors,' subproject 'Alimentation,' grant no $91.00226 . \mathrm{PF} 41$.

1 Wilmore DW. The metabolic management of the critically ill. New York: Plenum Press, 1977.

2 Rees RGP, Cooper TM, Beetham R, Frost PG, Silk BA. Influence of energy and nitrogen contents of enteral diets on nitrogen balance: a double blind prospective controlled clinical trial. Gut 1989; 30: 123-9.

3 Ziegler F, Ollivier JM, Cynober L, et al. Efficiency of enteral nitrogen support in surgical patients: small peptides vs nondegraded proteins. Gut 1990; 31: 1277-83.

4 Peuchant E, Salles C, Jensen R. Value of a spectroscopic 'fecalogram' in determining the etiology of steatorrhea. Clin Chem 1988; 34: 5-8.

5 Benini L, Caliari S, Guidi GC, et al. Near infrared spectrometry for faecal fat measurement: comparison with conventional gravimetric and titrimetric methods. Gut 1989; 30: 13447 .

6 Wetzel D. Near-infrared reflectance analysis. Sleeper among spectroscopic techniques. Anal Chem 1983; 55: 1165-71A.
7 Mark H, Workman J. Effect of repack on calibrations produced for near-infrared reflectance analysis. Anal Chem 1986; 58 : 145+-9.

8 Archibald RM Nitrogen by the Kjeldahl method. Stand Methods Clin Chem 1958; 2:91-9.

9. DiMagno EP, Clain JE Chronic pancrearis In: (jo VIW. Gardner JD, Brooks FP, I, ebenthal E, DiMagno EP, Scheele GA, eds. The exocrine pancreas. New York: Raven

10 Fairfull-Smith R, Abunassar R, Freeman JB, Maroun JA Rational use of elemental and non elemental diets in hospitalised patients. Ann Surg 1980; 192: 600-3

11 Andersson H, Bosaeus I, Ellegard L, Hallgren B, Hulten I., Magnusson O. Comparison of an elemental and two polymeric diets in colectomized patients with and without intestinal resection. Clin Nutr 1984; 3: 183-9.

12 Alpers DH, Rosenberg IH. Eating behaviour and nutrient requirements. In: Sleisenger MH, Fordtran JS, eds. (iastrointestinal disease. Philadelphia: WB Saunders, 1989: 19942006.

13 Grimble GK, West MFE, Acuti ABC, et al. Assessment of an automated chemiluminescence nitrogen analyzer for routine use in clinical nutrition. 7 Parenter Enteral Nutr 1988; 12: $100-6$ 